\title{
Correction: Capacity mechanisms: More flexibility reduces cost
}

Alessandro Rubino

Nature Energy 1, 16197 (2016); published 6 December 2016; corrected 23 January 2017.

In the version of this Research Highlight originally published, the image credit was missing. The image should have been credited to ollo/Getty Images. This error has been corrected in all versions of the Research Highlight. 